\title{
Impact of Comorbidities on Outcome in Children With COVID-19 at a Tertiary Care Pediatric Hospital
}

\author{
Dipti Kapoor, Virendra Kumar, Harish Pemde, Preeti Singh \\ From Department of Pediatrics, Lady Hardinge Medical College, New Delhi.
}

Correspondence to: Dr Dipti Kapoor, Associate Professor,

Department of Pediatrics, Lady Hardinge Medical College, New Delhi 110001.

diptikumar81@yahoo.co.in

Received: February 09, 2021;

Initial review: March 09, 2021;

Accepted: May 14, 2021.
Objective: To study the various comorbidities and their impact on outcome of severe acute respiratory syndrome coronavirus 2 (SARS-CoV-2) infected children. Methodology: Review of medical records of 120 children (58.4\% males), aged 1 month to 18 years, admitted between 1 March and 31 December, 2020 with at least one positive RT-PCR test for SARS-CoV-2. Clinical and demographic variables were compared between children with and without co-morbidities. Results: $62(51.7 \%)$ children had comorbidities. The most common comorbidity was tuberculosis $(32.3 \%)$ followed by other infections $(27.4 \%)$ and hematological $(19.4 \%)$ conditions. Fever $(89.2 \%)$ was the most common clinical feature followed by respiratory $(52.5 \%)$ and gastrointestinal $(32.5 \%)$ manifestations. There was no significant difference in the severity of COVID illness, length of hospital stay and adverse outcomes (ventilation and mortality) among children with and without comorbidities. Conclusion: The presence of a comorbid illness in pediatric inpatients with COVID-19 did not impact the illness severity, length of hospitalization, ventilation requirement and mortality.

Keywords: Mortality, Outcome, Tuberculosis, Ventilation.
$\mathrm{T}$ he Severe Acute Respiratory Syndrome Coronavirus-2 (SARS-CoV-2) pandemic has evolved rapidly leading to a multitude of presentations and variable severity, with substantial information regarding clinical manifestations and outcomes of coronavirus disease (COVID-19) in adults. It has been observed that presence of comorbidities is associated with severe illness and worse outcomes in adults infected with SARS-CoV-2 [1,2], but our knowledge about clinical characteristics as well as outcomes of COVID-19 infected children with comorbidities is limited. Moreover, there is limited literature on the spectrum of pediatric comorbidities and their outcome in association with SARS-CoV-2 infection from our country, which tends to be entirely different from those observed in the children from developed countries [3]. This study was planned to examine the effect of comorbidities with regard to disease presentation, evolution and outcomes in children infected with SARS-CoV-2.

\section{METHODS}

This case record review was undertaken at a tertiary care pediatric teaching hospital in northern India. During the SARS-CoV-2 outbreak, any child brought with history of recent onset fever, cough and/or fast breathing or other suggestive symptoms like recent onset fever with diarrhea or contact with COVID-19 positive patient was tested with RT-PCR test for SARS-CoV-2. These children were also screened for presence of any comorbidity. Comorbidity was defined as any distinct additional acute or chronic condition that has existed or may occur during the clinical course of a patient who has the index disease under study, and might alter the course of disease or the outcome [4].

Based on the results of confirmatory RT-PCR and clinical assessment, cases were classified as asymptomatic, mild, moderate and severe as per standard guidelines [5]. The criteria for admission for suspected COVID-19 illness included any of the following: respiratory distress, $\mathrm{SpO} 2$ on room air $<94$, shock/poor peripheral perfusion, poor oral intake or lethargy, specifically in infants and young children and/ or presence of seizures or encephalopathy [6]. Based on the results of confirmatory RT-PCR and clinical assessment, hospital treatment or home isolation measures were instituted with contact tracing measures as applicable (in accordance with the local prevailing guidelines). The patients were managed as per the standard WHO protocol [5].

All children 1 month to 18 years of age, with at least one positive RT-PCR test for SARS-CoV-2 and requiring admission between 1 March and 31 December, 2020 were 
included in the study. A special COVID ward was created during the ongoing pandemic for care of these children. Epidemiological, demographic, clinical, treatment, and outcome data of children with and without comorbidities was extracted from the case records and compared. The study was reviewed and approved by the institutional ethical committee with a waiver of consent for data collection.

Statistical analysis: Comparison of means between the two groups i.e., children with and without comorbidities was performed using the two-sample Student t-test. Categorical data were compared using Chi-square test. All tests were 2-tailed with the threshold level of significance at $P<0.05$. Statistical analysis was performed using STATA 14.2.

\section{RESULTS}

A total of 3180 suspected children were tested for SARSCoV-2; 295 (9.27\%) children tested positive. Amongst the latter, 120 SARS-CoV-2 positive children (70 boys)

Table I Comorbidities in SAR-CoV-2 Positive Pediatric Inpatients

\begin{tabular}{|c|c|c|}
\hline Comorbidity & $\begin{array}{l}\text { Disease } \\
n=62\end{array}$ & $\begin{array}{r}\text { Deaths } \\
n=12 \\
\end{array}$ \\
\hline Hematological ${ }^{a}$ & $12(19.4)$ & $2(16.7)$ \\
\hline Aplastic anemia & 2 & 1 \\
\hline Acute lymphoblastic leukemia & 7 & 1 \\
\hline Tuberculosis ${ }^{b}$ & $20(32.2)$ & $4(33.3)$ \\
\hline Disseminated & 9 & 3 \\
\hline Pulmonary & 7 & 1 \\
\hline Abdominal & 3 & 0 \\
\hline Renal & $5(8.1)$ & 0 \\
\hline Nephrotic syndrome & 4 & 0 \\
\hline Post-streptococcal glomerulonephriti & 1 & 0 \\
\hline Neurological & $5(8.1)$ & $1(8.3)$ \\
\hline Spastic cerebral palsy with epilepsy & 3 & 1 \\
\hline Insulin dependent diabetes mellitus & $1(1.6)$ & 0 \\
\hline Rheumatological $^{d}$ & $2(3.2)$ & $2(16.7)$ \\
\hline Surgical $^{e}$ & $2(3.2)$ & 0 \\
\hline Non-tubercular co-infections & $15(24.2)$ & $3(25)$ \\
\hline Disseminated staphylococcal sepsis & 4 & 2 \\
\hline Liver abscess & 7 & 0 \\
\hline Severe dengue & 4 & 1 \\
\hline
\end{tabular}

${ }^{{ }^{o} \text { one each with acute myeloid leukemia, Hodgkin disease and }}$ thalassemia; ${ }^{b}$ one with lymph node tuberculosis; ${ }^{c}$ one each with Tay Sachs disease and Gaucher disease; ${ }^{d}$ one each with systemic lupus erythromatosis and systemic onset juvenile idiopathic arthritis; ${ }^{e}$ one each with congenital cystic adenomatoid malformation and abdominal lump. required admission in the COVID ward. Fever $(89.2 \%)$ was the most common clinical feature at the time of presentation, and $64(53.3 \%)$ had severe acute malnutrition or thinness. Comorbidities were seen in $62(51.7 \%)$ children. The most common comorbidities were infections like tuberculosis (32.3\%) followed by other infections (27.4\%) (Table I).

The mortality rate in admitted patients was $24.2 \%$ $(n=29)$. There was no difference in the clinical characteristics of admitted children with and without comorbidities with respect to baseline characteristics. There was no statistical difference in the severity of COVID illness, mean duration of hospital stay and adverse outcomes like ventilation and mortality among the two groups (Table II). However, severe anemia and thrombocytopenia were present in significantly higher number of children with comorbidities (Table II).

Table II Characteristics of Coronavirus Disease 2019 (COVID-19) Patients With and Without Comorbidities

\begin{tabular}{|c|c|c|}
\hline Clinical parameter & $\begin{array}{l}\text { Comorbidity } \\
\text { present, }(n=62)\end{array}$ & $\begin{array}{r}\text { No comorbidity } \\
(n=58)\end{array}$ \\
\hline Mean age, $\mathrm{mo}^{a}$ & $58(34.6)$ & $62(28.4)$ \\
\hline Male & $38(61.2)$ & $32(55.1)$ \\
\hline Symptoms at admission & $n(\%)$ & $n(\%)$ \\
\hline Fever & $58(93.5)$ & $49(84.5)$ \\
\hline Respiratory & $34(54.9)$ & $29(50)$ \\
\hline Gastrointestinal & $22(35.5)$ & $17(29.3)$ \\
\hline Neurological & $3(4.8)$ & $4(6.9)$ \\
\hline Cardiovascular & $1(1.6)$ & $3(5.2)$ \\
\hline Others $^{b}$ & $2(3.2)$ & $5(8.6)$ \\
\hline SAM/severe thinness & $38(61.3)$ & $26(44.8)$ \\
\hline Hospital stay, (d) ${ }^{a}$ & $9.26(6.68)$ & $8.65(7.72)$ \\
\hline \multicolumn{3}{|l|}{ Severity of COVID illness } \\
\hline Mild to moderate & $46(74.2)$ & $38(65.5)$ \\
\hline Severe to critical & $16(25.8)$ & $20(34.5)$ \\
\hline Ventilated & $16(25.8)$ & $19(32.7)$ \\
\hline Deaths & $12(19.4)$ & $17(29.3)$ \\
\hline \multicolumn{3}{|l|}{ Laboratory parameters } \\
\hline Severe anemia ${ }^{c}$ & $21(33.9)$ & $9(15.5)$ \\
\hline Leucopenia $(<4000 /$ cu.mm) & $17(27.4)$ & $12(20.1)$ \\
\hline Lymphopenia $(<1500 /$ cu.mm) & $18 / 46(39.1)$ & $14 / 48(29.2)$ \\
\hline Thrombocytopenia $^{d}$ & $29(46.8)$ & $13(22.4)$ \\
\hline Elevated CRP $(>6 \mathrm{mg} / \mathrm{L})$ & $37(59.7)$ & $35(60.3)$ \\
\hline Elevated ESR $(>10 \mathrm{~mm} / \mathrm{h})$ & $29 / 39(74.3)$ & $16 / 23(69.6)$ \\
\hline
\end{tabular}

Values in no. (\%) or ${ }^{a_{m}}$ mean (SD). ${ }^{b}$ rash/myalgia/poor oral intake. SAM: severe acute malnutrition; CRP: C-reactive protein; ESR: erytherocyte sedimentation rate; ${ }^{c} P=0.02 ;{ }^{d} P=0.005$. 


\section{WHAT THIS STUDY ADDS?}

- Presence of a comorbid illness was not associated with increase in the severity of COVID illness, length of hospital stay or adverse outcome in children.

\section{DISCUSSION}

The clinical presentation in our cohort is similar to that observed by previous studies [7]. There was no significant difference in the clinical symptomatology, severity of COVID illness, mean duration of hospital stay and adverse outcomes among the children with and without comorbidities.

Tsankov, et al. [5] in their meta-analysis observed that the most common comorbidity in children infected with COVID-19 infection was obesity; whereas, we observed that more than $50 \%$ of our patients were underweight for age. The other comorbidities observed in their study were chronic respiratory conditions, cardiovascular disorders and neuromuscular diseases [5], whereas in our study where other infections were the most common comorbidities. The mortality rate observed in our study is higher than that reported globally [7], presumably due to referral bias due to our center being one of the largest tertiary care pediatric hospital in the public sector in this region.

There is a dearth of published literature on outcome of SARS-CoV-2 infected children with various comorbidities [8-11]. Three children with disseminated tuberculosis developed acute respiratory distress syndrome (ARDS) and multi-organ failure syndrome (MODS), and one died due to raised intracranial tension with neurogenic shock. The children with hematological disorders died secondary to febrile neutropenia with associated septicemia and catecholamine refractory shock. One child with spastic cerebral palsy was admitted with severe pneumonia and status epilepticus and went on to develop ARDS. The patient with systemic lupus erythematosus developed severe pneumonia with ARDS and acute kidney injury, whereas the patient with systemic onset juvenile idiopathic arthritis died due to macrophage activation syndrome and MODS. Two children died due to disseminated staphylococcal infection with catecholamine refractory shock, and one due to severe dengue with disseminated intravascular coagulation. The mortality was not significantly different across groups, possibly because most of our patients with comorbidities were under regular follow-up in our hospital and were well versed with the system, they might have presented early or might had been diagnosed early with symptoms of COVID-19 infection. Alternatively, some of these children were on immunomodulatory and immunosuppressant drugs, which could also have modified the course of infection by interfering with the cytokine storm responsible for organ damage in COVID-19 [12]. Most of the SARSCoV-2 infected children without comorbidities presented in advanced and decompensated clinical condition, presumably secondary to suboptimal management caused by delay in diagnosis, initiation of appropriate treatment, referral or transport during this unprecedented time of ongoing pandemic. However, this observation needs to be further evaluated in prospective studies with larger sample size.

Tsankov, et al. [5] in their meta-analysis also con-cluded that they could not determine whether comorbidities increase risk of severe COVID-19 in children. However, our observations are in contrast to those observed by Rao, et al. [13], who observed that presence of comorbidity increases the severity of COVID-19 disease.

Our study had limitations of having a retrospective design, small sample size and lack of follow-up. In spite of these shortcomings, this study provides preliminary data on the spectrum and outcome of comorbidities in children infected with SARS-CoV-2.

To conclude, the most common comorbidities observed in COVID infected children were infections like tuberculosis and other co-infections. There was no significant increase in the severity of COVID illness, duration of hospital stay or adverse outcome in these children. However, this observation does not understate the vulnerability of these children to develop severe illness and they should take all necessary precautions to avoid getting infected with SARS CoV-2. Further studies examining the effects of specific well-defined comorbidities are warranted to examine the effects that pediatric underlying conditions play in COVID-19 severity.

Ethical clearance: Institutional Ethical Committee, LH Medical College; No. LHMC/IEC/2020/97, dated November 6, 2020.

Contributors: DK: collected data and wrote the initial manuscript; VK, PK: critically analyzed the manuscript; PS: helped in data collection and revision of manuscript. All the authors read and approved the final manuscript.

Funding: None; Competing interests: None stated.

\section{REFERENCES}

1. Guan W, Liang W, Zhao Y, et al. Comorbidity and its impact on 1590 patients with COVID-19 in China: A nationwide analysis. European Respiratory J. 2020; 55: 
2000547

2. Zhou F, Yu T, Du R, et al. Clinical course and risk factors for mortality of adult in-patients with COVID-19 in Wuhan, China: A retrospective cohort study. The Lancet. 2020; 395:1054-62,

3. Tsankov BK, Allaire JM, Irvine MA, et al. Severe COVID19 Infection and pediatric comorbidities: A systematic review and meta-analysis. Intern J Infect Dis. 2021; 103: 246-56.

4. Valdres J M, Starfield b, Sibbald B, et al. Defining comorbidity: implications for understanding health and health services. Ann Fam Med. 2009; 7: 357-63.

5. Clinical management of severe acute respiratory infection when COVID-19 is suspected. Accessed October 31, 2020. Available at: https://www.who.int/publications-detail/ clinical-management-of-severe-acuterespiratory-infectionwhen-novel-coronavirus-(ncov)-infection-issuspected

6. Dong Y, Mo X, Hu Y, et al. Epidemiological characteristics of 2143 pediatric patients with 2019 coronavirus disease in China. Pediatrics. 2020; e20200702.

7. Meena J, Yadav J, Saini L, et al. Clinical features and outcome of SARS-CoV-2 infection in children: A syste- matic review and meta-analysis. Indian Pediatr. 2020; 57 : 820-26.

8. Shrinivasan R, Rane S, Pai M. India's syndemic of Tuberculosis and COVID-19. BMJ Global Health. 2020; 5: e003979.

9. Girmenia C, Gentile G, Micozzi A, et al. COVID-19 in patients with hematologic disorders undergoing therapy: Perspective of a large referral hematology center in Rome. Acta Haematol. 2020; 143: 574-82.

10. Adeiza SS, Shuaibu AB, Shuaibu GM. Random effects meta-analysis of COVID-19/S. Aureus partnership in coinfection GMS. Hygiene and Infection Control. 2020;15: 2196-5226.

11. Saddique A, Rana MS, Alam MM, et al. Emergence of coinfection of COVID-19 and dengue: A serious public health threat. J Infect. 2020;81:16-18.

12. Collange O, Tacquard C, Delabranche X, et al. Coronavirus disease 2019: associated multiple organ damage. Open Forum Infect Dis. 2020;7:249.

13. Rao S, Gavali V, Prabhu S, et al. Outcome of children admitted with SARS-CoV-2 infection: Experiences from a pediatric public hospital. Indian Pediatr. 2021;58:358-62. 